\title{
Fault Detection in Carrier Phase GPS Positioning Based on Hypotheses Testing of Innovation Processes
}

\author{
Yukihiro Kubo, Yoshiyuki Muto, Shinichi Kitao and Sueo Sugimoto \\ Dept. of Electrical and Electronic Engineering, Ritsumeikan University \\ 1-1-1, Noji-Higashi, Kusatsu City, Shiga 525-8577, Japan \\ E-mail: ykubo@se.ritsumei.ac.jp
}

\begin{abstract}
This paper proposes an anomalous measurement detection algorithm in carrier phase differential GPS(Global Positioning System) positioning in order to attain continuous precise positioning. In this paper, firstly the characteristics of the errors in the measurement data are briefly reviewed such that main error sources affecting the quality of the measurements are identified. Then a new algorithm which comprehensively detects these errors using the innovation process of the Kalman filter is presented.
\end{abstract}

\section{Introduction}

The quality of GPS positioning strongly depends on a number of factors. In order to attain continuous precise (sub-centimeter level accuracy) positioning results with carrier phase measurement, it is required that the integer ambiguity is correctly resolved, and errors unspecified as a functional or stochastic model have to be detected and efficiently corrected or removed. For data processing, cycle slips, receiver clock jumps, ionospheric, and multipath effects are the main sources that can deteriorate the quality of the measurements and the quality of the positioning. If there exist these errors, jumps and biases in the positioning results may appear.

There are some research activities focusing on these problems, e.g. [1, 2] for cycle slips, and [3] for multipath. For example, in [3], especially for the point positioning method, the multipath error detection algorithm is derived by applying the $\chi^{2}$-test of the innovation processes of the Kalman filter. In order to improve the detecting performance in real time kinematic (relative) positioning, this paper proposes an efficient algorithm by applying the $\chi^{2}$ and the likelihood ratio test.

In the proposed algorithm, in order to estimate the unknown values such as the position, velocity and ambiguity, the Kalman filter is applied and the cycle slip detection is implemented by using the combination of the likelihood ratio test and the $\chi^{2}$-test to the byproduct of the estimation; the innovation process of the Kalman filter[4].

\section{Double Difference Measurements}

We assume that two receivers $k$ and $u$ are located at the known point and the unknown point respectively, where the receiver $u$ is moving, namely kinematic positioning. When the receivers observe the waves from satellites $p$ and $q$, the integrated carrier phase measurements $\Phi_{L \mathrm{x}, k}^{p}, \Phi_{L \mathrm{x}, k}^{q}$ by the receiver $k$ and $\Phi_{L \mathrm{x}, u}^{p}$, $\Phi_{L \mathrm{x}, u}^{q}$ by $u$ are obtained, where $L \mathrm{x} ; \mathrm{x}=1,2$ indicates the frequency band such that $L 1=1575.42[\mathrm{MHz}]$ and $L 2=1227.60[\mathrm{MHz}]$ respectively. Then the double difference phase observation for $L \mathrm{x} ; \mathrm{x}=1,2$ wave is given by [5]

$$
\begin{aligned}
\Phi_{L \mathrm{x}, k u}^{p q}(t) \equiv & \left\{\Phi_{L \mathrm{x}, k}^{p}(t)-\Phi_{L \mathrm{x}, u}^{p}(t)\right\} \\
& -\left\{\Phi_{L \mathrm{x}, k}^{q}(t)-\Phi_{L \mathrm{x}, u}^{q}(t)\right\} \\
= & \left\{r_{k}^{p}(t)-r_{u}^{p}(t)\right\}-\left\{r_{k}^{q}(t)-r_{u}^{q}(t)\right\} \\
& -\delta I_{k u}^{p q}(t)+\delta T_{k u}^{p q}(t)+\delta m_{k u}^{p q}(t)+\delta s_{k u}^{p q}(t) \\
& +\lambda_{\mathrm{x}} N_{L \mathrm{x}, k u}^{p q}+\varepsilon_{L \mathrm{x}, k u}^{p q}(t) \\
\approx & \left\{r_{k}^{p}(t)-r_{u}^{p}(t)\right\}-\left\{r_{k}^{q}(t)-r_{u}^{q}(t)\right\} \\
& +\lambda_{\mathrm{x}} N_{L \mathrm{x}, k u}^{p q}+\varepsilon_{L \mathrm{x}, k u}^{p q}(t)
\end{aligned}
$$

where $\delta I_{k u}^{p q}$ and $\delta T_{k u}^{p q}$ denote ionospheric and tropospheric propagation delays respectively, $\delta m_{k u}^{p q}$ : multipath error, $\delta s_{k u}^{p q}$ : cycle slip and $\varepsilon_{L \mathrm{x}, k u}^{p q}$ : observation noise. Also $N_{L \mathrm{x}, k u}^{p q}$ is integer ambiguity, which is unknown integer constant at the initial time and $\lambda_{\mathrm{x}}$ is wave length. " $r$ " terms are the distance between the satellites and the receivers, for example, $r_{u}^{p}(t)$ denotes the distance between the satellite $p$ at the time $t-\tau_{u}^{p}$ and the receiver $u$ at the time $t$; where $\tau_{u}^{p}$ denotes the traveling time of the carrier wave from the satellite $p$ to the receiver $u$, namely,

$$
\begin{aligned}
r_{u}^{p}(t) & \equiv r_{u}^{p}\left(t, t-\tau_{u}^{p}\right) \\
& =\sqrt{\left(x_{u}-x^{p}\right)^{2}+\left(y_{u}-y^{p}\right)^{2}+\left(z_{u}-z^{p}\right)^{2}},
\end{aligned}
$$

where $\left(x_{u}, y_{u}, z_{u}\right)$ and $\left(x^{p}, y^{p}, z^{p}\right)$ are the coordinates of the receiver $u$ and the satellite $p$ in the WGS-84 frame [5,6], and time $t$ and $t-\tau_{u}^{p}$ are dropped from the receiver and the satellite coordinates respectively.

In the normal receiving condition, there exist no multipath and cycle slip, i.e.

$$
\delta m_{k u}^{p q}(t)=0, \quad \delta s_{k u}^{p q}(t)=0 .
$$


Also, we assume that two receivers located sufficiently close to each other (e. g. less than $10[\mathrm{~km}])$. Then ionospheric and tropospheric effects can be neglected[5]. Namely we assume

$$
\delta I_{k u}^{p q} \approx 0, \quad \delta T_{k u}^{p q} \approx 0 .
$$

Therefore Eq. (1) is the basic measurement equation for the kinematic positioning.

\subsection{System Model}

We consider here the kinematic positioning case such that the position of the receiver $k$ is known and the receiver $u$ is unknown (user) point. Denote $n_{s}$ as the number of satellite commonly observed by the receivers $k$ and $u$. We denote $p=1, q=2, \ldots, n_{s}$, then the measurement can be formed:

$$
\begin{aligned}
& \Phi_{L 1, k u}(i)=\left[\Phi_{L 1, k u}^{12}(i), \Phi_{L 1, k u}^{13}(i), \cdots, \Phi_{L 1, k u}^{1 n_{s}}(i)\right]^{\mathrm{T}}, \\
& \Phi_{L 2, k u}(i)=\left[\Phi_{L 2, k u}^{12}(i), \Phi_{L 2, k u}^{13}(i), \cdots, \Phi_{L 2, k u}^{1 n_{s}}(i)\right]^{\mathrm{T}},
\end{aligned}
$$

where $t=i \delta_{t}\left(\delta_{t}\right.$ is the data update interval of the receiver). In this kinematic positioning problem, we need to estimate three dimensional coordinates, velocities and accelerations defined by $x \equiv$ $\left[x_{u}, y_{u}, z_{u}\left|v_{x}, v_{y}, v_{z}\right| a_{x}, a_{y}, a_{z}\right]^{\mathrm{T}}$ for the unknown moving position and $2 \times\left(n_{s}-1\right)$ values:

$$
\begin{aligned}
N & \equiv\left[N_{L 1}^{\mathrm{T}}, N_{L 2}^{\mathrm{T}}\right]^{\mathrm{T}} \\
& =\left[N_{L 1, k u}^{12}, \cdots, N_{L 1, k u}^{1\left(n_{s}\right)}, N_{L 2, k u}^{12}, \cdots, N_{L 2, k u}^{1\left(n_{s}\right)}\right]^{\mathrm{T}},
\end{aligned}
$$

which is corresponding to the integer ambiguity in Eqs. (3) and (4). Therefore the state vector $\boldsymbol{\eta}$ consists of $9+2\left(n_{s}-1\right)=2 n_{s}+7$ components, as follows:

$$
\eta(i) \equiv\left[\boldsymbol{x}^{\mathrm{T}}(i), \boldsymbol{N}^{\mathrm{T}}(i)\right]^{\mathrm{T}} .
$$

Applying the dynamical models for the moving receiver $[7,8]$, we have the following state equation,

$$
\begin{gathered}
\boldsymbol{\eta}(i+1)=A(i) \boldsymbol{\eta}(i)+B(i) \boldsymbol{w}(i), \\
i=1,2, \ldots, n
\end{gathered}
$$

where $A$ and $B$ are known matrices with appropriate dimensions, $w(i)$ is the process noise, and $n$ is the number of available data.

From Eqs. (1)-(4), we can formulate the observation equation as follows:

$$
\tilde{y}(i)=\left[\begin{array}{l}
h(x(i)) \\
h(x(i))
\end{array}\right]+\left[\begin{array}{c}
\lambda_{1} N_{L 1} \\
\lambda_{2} N_{L 2}
\end{array}\right]+v(i),
$$

where $\boldsymbol{h}$ is a function derived from Eqs. (1)-(4), and

$$
\tilde{\boldsymbol{y}}(i) \equiv\left[\begin{array}{c}
\Phi_{L 1, k u}(i) \\
\Phi_{L 2, k u}(i)
\end{array}\right], \quad \boldsymbol{v}(i)=\left[\begin{array}{c}
\boldsymbol{v}_{L 1}(i) \\
\boldsymbol{v}_{L 2}(i)
\end{array}\right] .
$$

Also we assume that $\boldsymbol{v}_{L 1}(i)$ and $v_{L 2}(i)$ are mutually independent white Gaussian noises with their covariance matrices $R_{L 1}$ and $R_{L 2}$.

\subsection{Kalman Filtering}

The measurement Eq. (7) is nonlinear due to the square root terms in Eq. (2). Therefore the extended Kalman filter is applied. Thus $\boldsymbol{h}(\boldsymbol{x}(i))$ function is linearized by applying first order Taylor series approximation around the predictor $\hat{x}(i \mid i-1)$. Then from Eqs. (6) and (7), we have an approximated linear observation equation as

$$
\boldsymbol{y}(i)=C(i) \boldsymbol{\eta}(i)+\boldsymbol{v}(i),
$$

where $C(i)$ and $H(i)$ are given as follows:

$$
\begin{aligned}
C(i) & \equiv\left[\begin{array}{ccc}
H(i) & \lambda_{1} I & O \\
H(i) & O & \lambda_{2} I
\end{array}\right] \\
H(i) & \equiv\left[\frac{\partial \boldsymbol{h}(\boldsymbol{x}(i))}{\partial \boldsymbol{x}(i)}\right]_{\boldsymbol{x}(i)=\hat{\boldsymbol{x}}(i \mid i-1)}^{\mathrm{T}}
\end{aligned}
$$

By the extended Kalman filter, we can obtain the estimate of the position and ambiguity as $\hat{\eta}$. However, the estimate of the ambiguity $\hat{N}$ in $\hat{\boldsymbol{\eta}}$ is obtained as real numbers. Since $N$ is exactly integer, the LAMBDA method [9] is applied in order to obtain integer estimate from $\hat{N}$.

As byproduct of the estimation process, the innovation process $\nu(i)$ and its covariance matrix $M(i)$ are obtained as follows:

$$
\begin{aligned}
\boldsymbol{\nu}(i) & =\boldsymbol{y}(i)-C(i) \hat{\boldsymbol{\eta}}(i \mid i-1) \\
M(i) & =E\left\{\boldsymbol{\nu}(i) \boldsymbol{\nu}^{\mathrm{T}}(i)\right\} \\
& =C(i) P(i \mid i-1) C^{\mathrm{T}}(i)+R(i) \\
& \equiv\left[M_{j k}(i)\right], \quad j, k=1,2, \ldots, 2 n_{s}-2,
\end{aligned}
$$

where $P(i \mid i-1)$ is the error covariance matrix of the prediction $\hat{\eta}(i \mid i-1)$ of $\eta(i)$ which appears in the extended Kalman filtering process and $R(i)$ is the covariance matrix of the measurement noise $\boldsymbol{v}(i)$.

It is well known that under the regular condition, the innovation process $\nu(i)$ is a zero mean Gaussian white noise process with the covariance $M(i)$ [4].

\section{Detecting Anomalous Measurements}

In an anomalous receiving condition, some measurement errors may occur. As described in the previous section, in such case, the dominant error sources are multipath error due to wave refraction by buildings, cycle slips due to obstructions of the satellite and low SNR (Signal to Noise Ratio). The cycle slip $\delta s_{k u}^{p q}$ is a jump of integer number in the ambiguity $N[10,5]$. Moreover the ionospheric and tropospheric delays $\delta I_{k u}^{p q}$ and $\delta T_{k u}^{p q}$ may not be negligible due to changes in atmospheric condition. They cause changes in the information of the measurement that are unknown magnitude, and occur at unknown instants time $t$, consequently result in the degradation of the positioning accuracy. It 
is usually difficult to detect them by observing directly carrier phase data because sometimes they have small magnitude relative to the rate of phase increment. In this paper, we observe the change of the innovation process in extended Kalman filtering.

\subsection{Applying $\chi^{2}$ Test}

Now we apply the Cholesky factorization[11] to $M(i)$ as follows

$$
M(i)=L(i) L^{\mathrm{T}}(i),
$$

and define the process:

$$
\nu_{s}(i) \equiv L^{-1}(i) \nu(i) .
$$

Since the innovation process in (10) is a white Gaussian with the covarinace matrix $M(i)$ in Eq. (11), each element of $\nu_{s}(i)$ is statistically independent to other elements of $\nu_{s}(i)$ and each is a Gaussian process. Therefore the statistics $T_{c}(i)$ of the inner product of $\nu_{s}(i)$ follow to the $\chi^{2}$ distribution, with $2 n_{s}-2$ degree of freedom, namely,

$$
T_{c}(i) \equiv \nu_{s}(i)^{\mathrm{T}} \nu_{s}(i) .
$$

If an abrupt change in measurements occurs, the covariance matrix of the innovation process changes. Therefore, we formulate two hypotheses such as

$$
\begin{aligned}
& H_{\chi, 0}: \text { the change does not occur } \\
& H_{\chi, 1}: \text { the change occurs. }
\end{aligned}
$$

The decision is, therefore, based on $\chi^{2}$ test. The hypothesis $H_{\chi, 1}$ is accepted so that a certain statistical change occurs. Namely, our $\chi^{2}$ test is as follows: Decide the level of significance ( $\alpha=0.95,0.99$ etc.), then, accept the hypothesis $H_{\chi, 0}$ if

$$
T_{c}(i)<\chi_{1-\alpha}^{2}\left(2 n_{s}-2\right)
$$

or accept the hypothesis $H_{\chi, 1}$

$$
T_{c}(i)>\chi_{1-\alpha}^{2}\left(2 n_{s}-2\right) .
$$

\subsection{Applying Likelihood Ratio Test}

In the proposed method, also we apply the likelihood ratio (LR) test in order to detect the occurrence time of an abrupt change, and avoid type II error of $\chi^{2}$ test described in the previous section as far as possible.

The likelihood ratio test is done for each component of the innovation process. Namely define a scalar standard innovation process

$$
\nu_{s, j}(i) \equiv \sqrt{\frac{1}{M_{j j}(i)}} \nu_{j}(i), \quad j=1, \cdots, 2 n_{s}-2 .
$$

Then it is shown that $\nu_{s, j}(i) \sim \mathcal{N}(0,1)$. Therefore the joint probability density of $\left\{\nu_{s, j}(k), k=1, \cdots, i\right\}$ is given by

$$
p\left(\nu_{s, j}(1), \cdots, \nu_{s, j}(i)\right)=\prod_{k=1}^{i} \frac{1}{\sqrt{2 \pi}} \exp \left\{-\frac{1}{2} \nu_{s, j}^{2}(k)\right\} .
$$

If an abrupt change in the measurement occurs at time $i \doteq r$, then the mean and covariance of the standard innovation process changes from 0 to an unknown value $\mu_{j}$ and to an unknown covariance $\sigma_{j}^{2}$, respectively; namely

$$
\begin{gathered}
E\left\{\nu_{s, j}(k)\right\}= \begin{cases}0 & (1 \leq k \leq r-1) \\
\mu_{j} & (r \leq k \leq i),\end{cases} \\
\operatorname{Var}\left\{\nu_{s, j}(k)\right\}= \begin{cases}1 & (1 \leq k \leq r-1) \\
\sigma_{j}^{2} & (r \leq k \leq i) .\end{cases}
\end{gathered}
$$

Therefore, we formulate again two hypotheses such that

$H_{L, 0}$ : the change does not occur upto the time $i$

$H_{L, 1}$ : the change occurs at time $r \leq i$.

The LR between these two hypotheses is

$$
\prod_{k=r}^{i} \frac{p_{1}\left(\nu_{s, j}(k)\right)}{p_{0}\left(\nu_{s, j}(k)\right)}
$$

Namely, for Eqs. (17) and (18) we have

$$
\prod_{k=r}^{i} \frac{1}{\sqrt{\sigma_{j}^{2}}} \exp \left\{\frac{\nu_{s, j}^{2}(k)}{2}-\frac{\left(\nu_{s, j}(k)-\mu_{j}\right)^{2}}{2 \sigma_{j}^{2}}\right\} \text {. }
$$

Then the log-likelihood ratio is given by

$$
\begin{array}{r}
l_{j, i}\left(r, \mu_{j}, \sigma_{j}^{2}\right)=\frac{1}{2} \sum_{k=r}^{i}\left[-\ln \sigma_{j}^{2}+\nu_{s, j}^{2}(k)\right. \\
\left.-\frac{\left(\nu_{s, j}(k)-\mu_{j}\right)^{2}}{2 \sigma_{s, j}^{2}}\right] .
\end{array}
$$

Replacing the unknown changed time $r$ by its maximum likelihood estimate (MLE) under $H_{L, 1}$, namely

$$
\hat{r}(i)=\underset{1 \leq r \leq i}{\arg \max } l_{j, i}\left(r, \hat{\mu}_{j}, \hat{\sigma}_{j}^{2}\right),
$$

where $\hat{\mu}_{j}$ and $\hat{\sigma}_{j}^{2}$ are MLEs of $\mu_{j}$ and $\sigma_{j}^{2}$, respectively, which are given by

$$
\begin{aligned}
\hat{\mu}_{j}(i) & =\frac{1}{i-r+1} \sum_{k=r}^{i} \nu_{s, j}(k), \\
\hat{\sigma}_{j}^{2} & =\frac{1}{i-r+1} \sum_{k=r}^{i}\left(\nu_{s, j}(k)-\hat{\mu}_{j}\right)^{2} .
\end{aligned}
$$

Then the $\log \mathrm{LR}$ is given by

$$
\begin{aligned}
l_{j, i}\left(r, \hat{\mu}_{j}, \hat{\sigma}_{j}^{2}\right) & \\
\quad= & \frac{1}{2} \sum_{k=r}^{i}\left[-\ln \sigma_{j}^{2}+\nu_{s, j}^{2}(k)-\frac{\left(\nu_{s, j}(k)-\mu_{j}\right)^{2}}{\sigma_{s, j}^{2}}\right] \\
\quad= & \frac{1}{2}\left[-(i-r+1)\left(\ln \hat{\sigma}_{j}^{2}+1\right)+\sum_{k=r}^{i} \nu_{s, j}^{2}(k)\right] .
\end{aligned}
$$


Finally we obtain the following change detector,

$$
\begin{aligned}
g_{j}(i) \equiv & \max _{1 \leq r \leq i} l_{j, i}\left(r, \hat{\mu}_{j}, \hat{\sigma}_{j}^{2}\right) \\
= & \max _{1 \leq r \leq i} \sum_{k=r}^{i}\left[-\frac{n_{s}-1}{2} \ln \hat{\sigma}_{\nu_{s}}^{2}+\frac{1}{2} \nu_{s}^{\mathrm{T}}(k) \nu_{s}(k)\right. \\
& \left.-\frac{1}{2 \sigma_{\nu_{s}}^{2}}\left(\nu_{s}(k)-\hat{\mu}\right)^{\mathrm{T}}\left(\nu_{s}(k)-\hat{\mu}\right)\right] .
\end{aligned}
$$

If $g_{j}(i)$ is greater than threshold $\lambda_{g}$, hypothesis $H_{L, 1}$ is accepted with changing time $\hat{r}$, otherwise $H_{L, 0}$ is accepted.

\section{Experimental Results}

The experiment of the presented anomalous measurement detection and positioning algorithm has been carried out. In the experiment, two receivers which composed GPS-600 antenna and MiLLenium GPSCard (OEM3) of NovAtel Inc. were located at the known position (rooftop of a 7-story building ) and the unknown position of a moving land-vehicle respectively in Ritsumeikan University. The land-vehicle moved around a 400 meters athletic track. The baseline length was about $200 \sim 300[\mathrm{~m}]$. A total of 4558 epochs dual frequency data were collected at one second interval on June 6, 2002, from 4:42 ' 23 to 5:58 ' 20 UTC, where approximately the first 18 minutes data were collected in the static situation, i.e. the land-vehicle stopped at the starting point in order to obtain precise coordinate of the point, and the last 30 minutes data were collected in the kinematic situation.

Firstly, by using the data in the static period, i.e. from 1st to 1057th epoch (4:42 ' 23 to 5:00 '0 UTC) and all 10 satellites in view, the static positioning was carried out and the integer ambiguities were obtained. They were regarded as true in evaluating the results of the following kinematic experiments.

For implementing the Kalman filter, so-called Singer model[7] was applied as the dynamical model of the vehicle. Some parameters and initial value were given as follows. The initial estimates of the integer ambiguity and the coordinate were obtained from the double differences of the pseudo range measurements[5]. According to [7], matrices $A$ and $B$ in Eq. (6) were determined from some parameters such as the reciprocal of the acceleration time constant: 1.01 , the standard deviation of the acceleration: 0.36 and the system noise variance of the ambiguity: $1 \times 10^{-3}$. They were determined experimentally. Also the standard deviation of the measurement noise $\varepsilon_{L x, k u}^{p q}$ was $0.19[\mathrm{~cm}][5]$. The land-vehicle started to move, and the kinematic experiments were carried out by using the data from 2757 th to 4557th epoch (5:28 ' 20 to 5:58 '20) UTC and PRNs 24, $10,8,4,23$ satellites, where the PRN 24 was selected as the primary satellite for the computation of the double difference observations. Throughout the experiment,

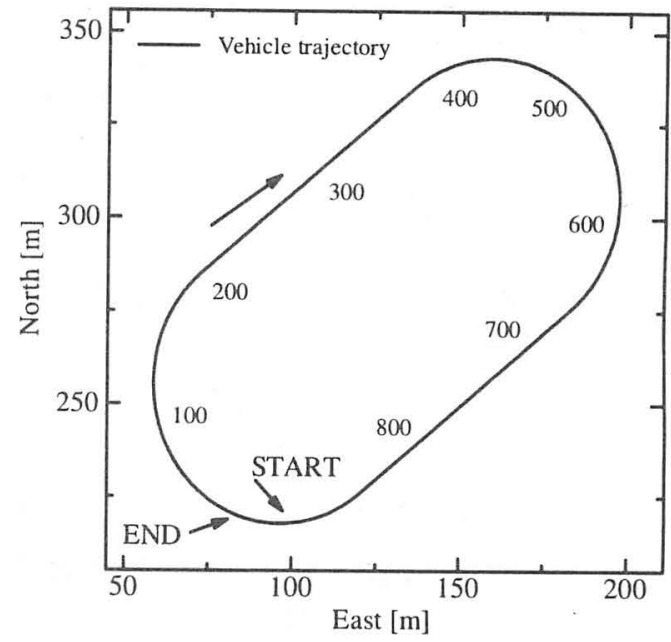

Fig. 1: Vehicle trajectory without cycle slips
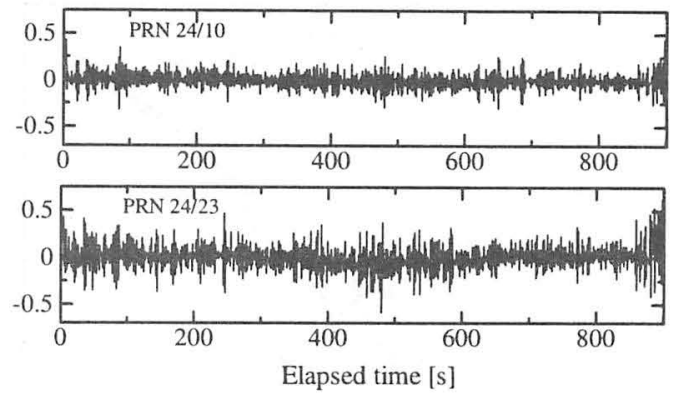

Fig. 2: Innovation processes without cycle slips

therefore, total 8 double difference observations were available at each epoch. After starting to move, our kinematic positioning algorithm correctly resolved the ambiguity in several epochs.

\subsection{Results of Normal Condition}

Fig. 1 shows the trajectory of estimated land-vehicle position. And Fig. 2 shows two innovation processes of satellite pairs PRNs 24, 10 and 24, 23 partially extracted from total 8 innovations. We can see clearly from Figs. 1 and 2 that the land-vehicle moved around the athletic track and no abrupt change in measurement occurred.

\subsection{Cycle Slip Case}

Next, we examined the positioning and detecting performance under an anomalous situation. The test was implemented with an artificial cycle slip caused by

Table 1: An example of simulated cycle slips

\begin{tabular}{|l|c|c|c|c|}
\hline & \multicolumn{4}{|c|}{ Satellite pair of double difference } \\
\cline { 2 - 5 } & $(24,10)$ & $(24,23)$ & $(24,8)$ & $(24,6)$ \\
\hline L1 cycle slips & 0 & +3 & 0 & 0 \\
\hline L2 cycle slips & 0 & 0 & 0 & 0 \\
\hline
\end{tabular}




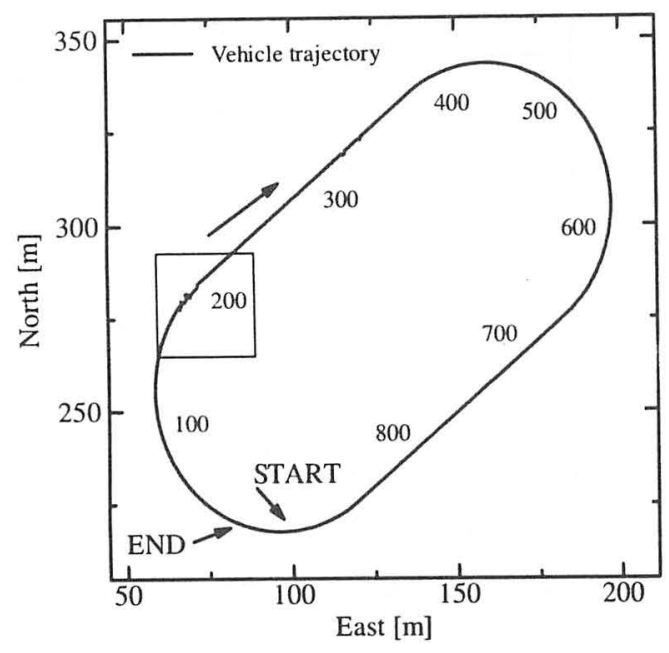

Fig. 3: Vehicle trajectory with cycle slip (No correction)

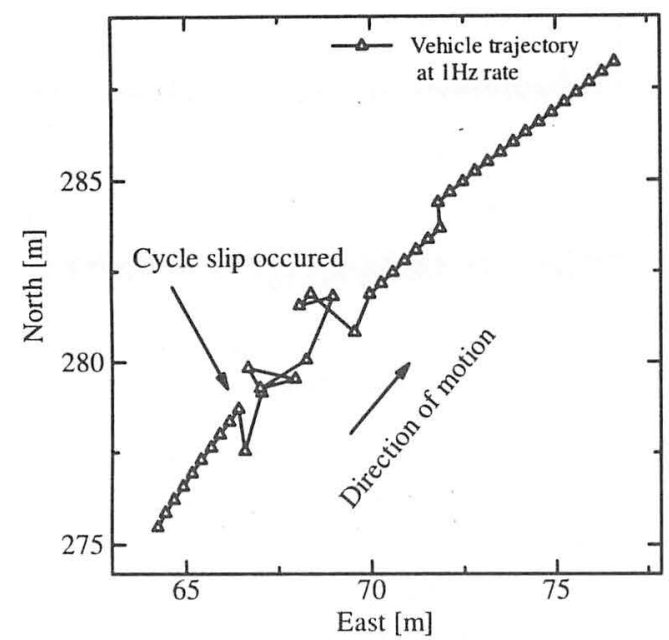

Fig. 4: Enlarged view of rectangular region of Fig. 3

adding randomly selected integer number to the original carrier phase observation data described above. A typical example of such situations is shown in Table 1 , i.e. the value of " +3 " cycle slip occurred at 200 th epoch in the L1 double differenced data of PRNs 24 and 23. In other words, there exist " +3 " cycle slip in the L1 wave from satellite PRN 23. Fig. 3 shows the estimated vehicle trajectory with no cycle slip correction and Fig. 4 shows the enlarged view of the rectangular region indicated in Fig. 3. In this region, we can see that the positioning performance is seriously degraded due to the cycle slip.

In this experiment, the $\chi^{2}$ test of Eqs. (13) and (14) and the component wise likelihood ratio test were applied to detect the cycle slip. Fig. 6 and 7 show the test statistics, that is, the likelihood ratio of PRNs 24, 10 and 24,23 , and the sum of squared orthogonalized innovation that were respectively obtained from Eqs. (26) and (12) based on the innovation processes partially shown in Fig. 5. The thresholds were 4.0 for the likeli-
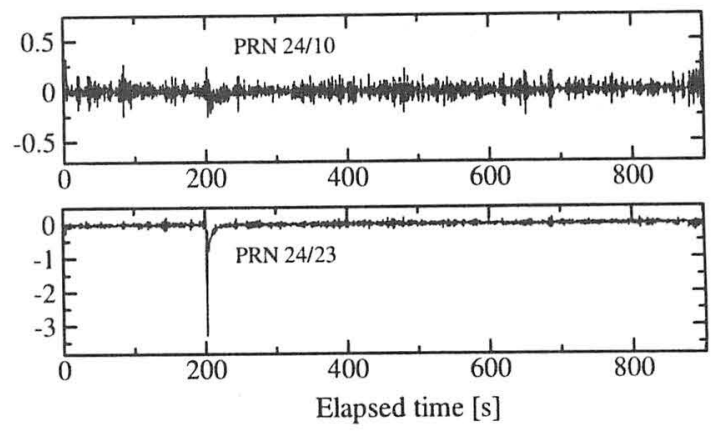

Fig. 5: Innovation processes with cycle slips

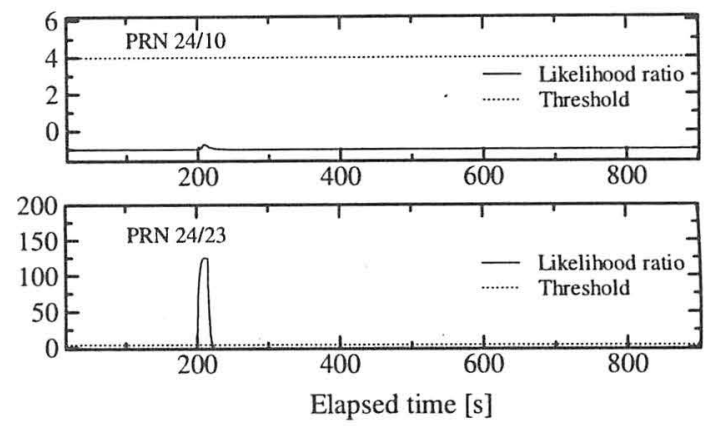

Fig. 6: Likelihood ratio

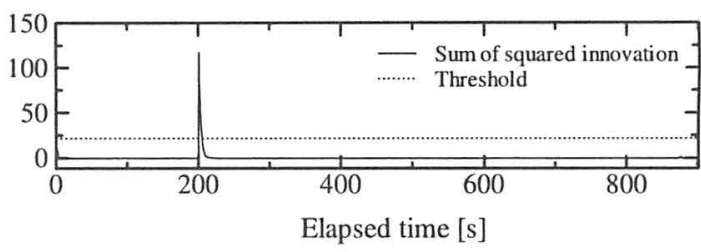

Fig. 7: Sum of squared innovation

hood ratio test in Fig. 6, and 22.0 for the $\chi^{2}$ test (level of significance $\alpha=0.005$ ) in Fig. 7. In this case, the algorithm presented in this paper could correctly detect the cycle slip at 200th epoch.

The error source considered in this experiment is cycle slip. And if they are correctly detected, there are possibilities of removing or correcting them. Therefore the cycle slip correction procedure of [12] was implemented, i.e. the ambiguity in the state vector was reset to the value that was obtained from the position estimate of the Kalman filter and the double difference observation at that epoch. Fig. 8 shows the trajectory of the vehicle obtained by correcting the cycle slip. Fig. 9 shows the enlarged view of the rectangular region indicated in Fig. 8. And Fig. 10 shows the corrected innovation processes. From Figs. 8 to 10, we can observe that the presented algorithm could correctly detected the cycle slip and rapidly (within several epochs) correct it. 


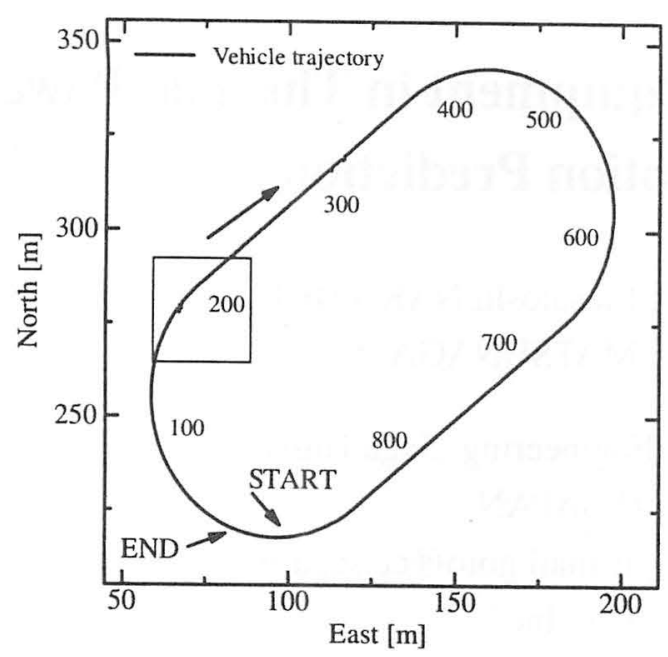

Fig. 8: Vehicle trajectory with cycle slip correction

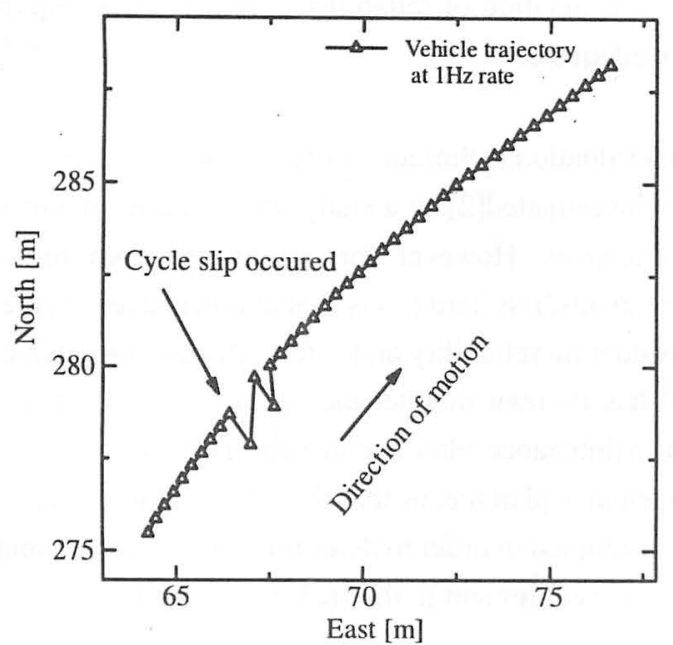

Fig. 9: Enlarged view of rectangular region of Fig. 8

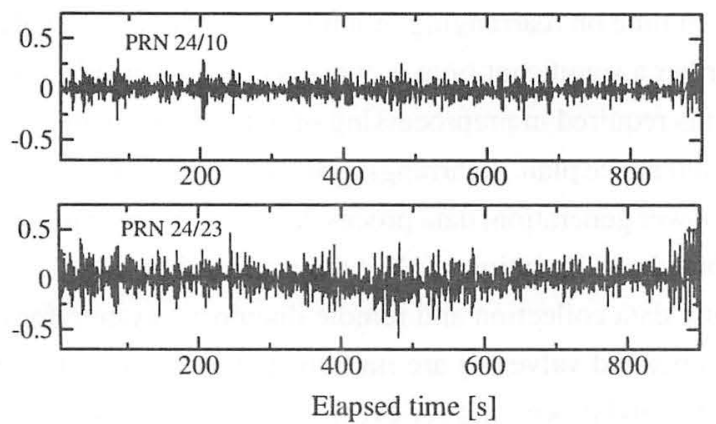

Fig. 10: Innovation processes with cycle slip correction

\section{Conclusion}

In this paper, the algorithm with cycle slips detection and correction by using the combination of $\chi^{2}$ and LR tests has been proposed. The algorithm is also efficiently combined with the positioning algorithm that are applied by the Kalman filter and the LAMBDA method. Examinations of the algorithm were done by using real receiver data and artificially simulated cycle slip. The experimental results show that the algorithm in this paper can detect and correct cycle slips efficiently, and it provides better positioning accuracy. In this paper, the cycle slips have been the subject of our experiment, however we expect this algorithm also work well for detecting other abrupt measurement for example residual ionospheric and tropospheric effects and multipath.

\section{References}

[1] S. P. Mertikas and C. Rizos: On-line Detection of Abrupt Changes in the Carrier-Phase Measurements of GPS, Journal of Geodesy, Vol. 71, pp. 469-482, 1997.

[2] D. Kim and R. B. Langley: Instantaneous RealTime Cycle-Slip Correction for Quality Control of GPS Carrier-Phase Measurements, Navigation: J. Institute of Navigation, Vol. 49, No. 4, Winter, pp. 205-222, 2002-2003.

[3] H. K. Lee, J. G. Lee and G.-I. Jee: GPS Multipath Detection Based on Sequence of SuccessiveTime Double-Differences, IEEE Signal Processing Letters, Vol. 11, No. 3, pp. 316-319, 3, 2004.

[4] A. Gelb(ed.): Applied Optimal Estimation, MIT Press, Massachusetts, 1974.

[5] B. W. Parkinson and J. J. Spilker Jr.(Eds.): Global Positioning System: Theory and Applications, Vol. I, II, AIAA, Washington, DC, 1997.

[6] S. Sugimoto, Y. Kubo and H. Kumagai: GPS Navigation Algorithms and Estimation - Detection Theory, Systems, Control and Information ISCIE, Vol. 46, No. 5, pp. 276-285, 2002 (in Japanese).

[7] R. A. Singer: Estimating Optimal Tracking Filter Performance for Manned Maneuvering Targets, IEEE Trans. Aerospace and Electronic Systems, Vol. AES-6, No. 4, pp. 197-204, July, 1970.

[8] C. Uratani, K. Sone, Y. Muto, S. Maruo and S. Sugimoto: Dynamical Models for Carrier-Phase Kinematic GPS Positioning, Proc. of the ION GPS/GNSS 2003, pp. 809-818, Portland, 2003.

[9] P. J. G. Teunissen: The Least-Squares Ambiguity Decorrelation Adjustment: A Method for Fast GPS Integer Ambiguity Estimation, J. Geodesy, Vol. 70, pp. 65-82, 1995.

[10] A. Leick: GPS Satellite Surveying, Johon Wiley \& Sons, New York, 1990.

[11] G. H. Golub and C. F. V. Loan: Matrix Computations, Second Edition, Johns Hopkins University Press, London, 1989.

[12] Y. Kubo, S. Maruo and S. Sugimoto: Kalman Filtering Approach for Integer Ambiguity Resolution in GPS Positioning with Varying Visible Satellites, Proc. of IASTED International Conference on Signal and Image Processing, pp. 420-425, Hawaii, Aug., 2001. 